\title{
LINEAR METHODS WHICH SUM SEQUENCES OF BOUNDED VARIATION
}

\author{
DAVID F. DAWSON
}

A complex sequence $\left\{z_{p}\right\}$ is said to be of bounded variation provided $\sum\left|z_{p}-z_{p+1}\right|<\infty$. In this paper we show that a matrix which sums every sequence of bounded variation also sums a convergent sequence not of bounded variation (Theorem 1). Indeed, if $M$ is a countable set of matrices, each of which sums every sequence of bounded variation, then there is a convergent sequence not of bounded variation which every matrix in $M$ sums (Theorem 2). Our proofs are by direct construction. We are indebted to the referee for the following observation: Theorem 1 follows from a rather inaccessible result of Mazur-Orlicz-Zeller (see p. 125 of [4] and p. 256 of [5]) to the effect that the set of all convergent sequences which a matrix sums, as an $F K$ space, has a separable dual space, while the space of sequences of bounded variation does not, since its maximal subspace of null sequences is equivalent to $\left\{z: \sum\left|z_{i}\right|<\infty\right\}$ whose dual is the set of all bounded sequences.

A basic tool in this study is the fact [1], [3] that a matrix $\left(a_{p q}\right)$ sums every sequence of bounded variation if and only if

(i) $\left\{a_{p n}\right\}_{p=1}^{\infty}$ converges, $\quad n=1,2,3, \cdots$,

(ii) $\left\{\sum_{p=1}^{\infty} a_{n p}\right\}_{n=1}^{\infty}$ converges, and

(iii) there exists $k$ such that $\left|\sum_{p=1}^{j} a_{n p}\right|<k, \quad n, j \geqq 1$.

We will denote the set of all convergent complex sequences by $S_{C}$ and the set of all complex sequences of bounded variation by $S_{B V}$. Throughout, we will use the notation $x=\left\{x_{p}\right\}$, and, given a sequence $y$ and an increasing sequence $\alpha$ of positive integers, we will use $F(y, \alpha)$ to denote the sequence $z$ such that for each positive integer $j$, $z_{p}=y_{j}, \alpha_{j-1}<p \leqq \alpha_{j},\left(\alpha_{0}=0\right)$.

Lemma. If $\sum a_{p}$ has bounded partial sums $\left\{s_{p}\right\}$, then there exists an increasing sequence $\alpha$ of positive integers such that if $y$ is a null sequence and $F(y, \alpha)=x$, then $\sum x_{p} a_{p}$ converges.

Received by the editors June 12, 1965. 
Proof. Let $\alpha$ be an increasing sequence of positive integers such that $\left\{s_{\alpha_{p}}\right\} \in S_{B V}$. Let $y$ be a null sequence and $F(y, \alpha)=x$. Suppose $k>\alpha_{1}$ is an integer and $t$ is the largest integer such that $\alpha_{t}<k$. Then, using summation by parts, we have

$$
\sum_{p=1}^{k} x_{p} a_{p}=\sum_{p=1}^{t-1} s_{\alpha_{p}}\left(x_{\alpha_{p}}-x_{\alpha_{p}+1}\right)+s_{\alpha_{t}} x_{\alpha_{t}}+\sum_{p=\alpha_{t}+1}^{k} x_{p} a_{p} .
$$

By a well-known theorem of Hadamard [2], $\sum_{p=1}^{\infty} s_{\alpha_{p}}\left(x_{\alpha_{p}}-x_{\alpha_{p}+1}\right)$ converges since $\sum_{p=1}^{\infty}\left(x_{\alpha_{p}}-x_{\alpha_{p}+1}\right)=\sum_{p=1}^{\infty}\left(y_{p}-y_{p+1}\right)$ converges and $\left\{s_{\alpha_{p}}\right\}_{p=1}^{\infty}$ converges absolutely, i.e., is of bounded variation. Thus from (2) we see that $\sum x_{p} a_{p}$ converges since $\left\{s_{p}\right\}$ is bounded, $x$ and $y$ are null sequences, and the last term on the right side of (2) is equal to $y_{t+1}\left(s_{k}-s_{\alpha_{t}}\right)$. This completes the proof.

REMARK 1. Suppose $\sum a_{p}$ has bounded partial sums $\left\{s_{p}\right\}$ and $\alpha$ is an increasing sequence of positive integers such that $\left\{s_{\alpha_{p}}\right\} \in S_{B V}$. It is clear from the proof of the lemma that if $\alpha^{\prime}$ is an increasing sequence of positive integers such that for some $m,\left\{\alpha_{p}^{\prime}\right\}_{p=m}^{\infty}$ is a subsequence of $\alpha, y$ is a null sequence, and $F\left(y, \alpha^{\prime}\right)=x$, then $\sum x_{p} a_{p}$ converges.

Theorem 1. If $A$ is a matrix which sums every sequence in $S_{B V}$, then there exists $x \in S_{C}-S_{B V}$ such that $A x \in S_{C}$.

Proof. Suppose $A=\left(a_{p q}\right)$ sums every sequence in $S_{B V}$. Using (i) of (1), we let $a_{n}=\lim _{p \rightarrow \infty} a_{p n}$, and using $\left\{s_{p}\right\}$ for the partial sums of $\sum a_{p}$, we note that (i) and (iii) of (1) can be used to show that $\left|s_{p}\right| \leqq k$ for all $p \geqq 1$. Let $\alpha$ be an increasing sequence of positive integers such that $\left\{s_{\alpha_{p}}\right\} \in S_{B V}$. Let $t_{1}$ be a positive integer such that if $q \geqq t_{1}$, then $\left|a_{q i}-a_{i}\right|<1 / 2^{i+1}, i=1,2, \cdots, \alpha_{1}$. Let $r_{1}=\alpha_{1}$ and let $r_{2}$ be the first term of $\alpha$ following $\alpha_{1}$ such that if $j$ is a positive integer, then $\left|\sum_{q=\tau_{2}+j}^{\infty} a_{p q}\right|<1 / 2^{2}, p=1,2, \cdots, t_{1}$. Let $t_{2}$ be an integer greater than $t_{1}$ such that if $q \geqq t_{2}$, then $\left|a_{q i}-a_{i}\right|<1 / 2^{i+2}, i=1,2, \cdots, r_{2}$. Let $r_{3}$ be the first term of $\alpha$ following $r_{2}$ such that if $j$ is a positive integer, then $\left|\sum_{q=r_{s}+1}^{\infty} a_{p q}\right|<1 / 2^{3}, p=1,2, \cdots, t_{2}$. We continue the process to obtain sequences $\left\{r_{p}\right\}$ and $\left\{t_{p}\right\}$ such that $\left\{r_{p}\right\}$ is a subsequence of $\alpha,\left\{t_{p}\right\}$ is an increasing sequence of positive integers, and if $m$ if a positive integer, then for $q \geqq t_{m}$,

$$
\left|a_{q i}-a_{i}\right|<1 / 2^{i+m}, \quad i=1,2, \cdots, r_{m},
$$

and if $j$ is a positive integer and $m>1$, then

$$
\left|\sum_{q=r_{m}+j}^{\infty} a_{p q}\right|<1 / 2^{m}, \quad p=1,2, \cdots, t_{m-1} .
$$


Suppose $y$ is a null sequence and let $F(y, r)=x$. By using (4), we can easily show that if $n$ is a positive integer, then $\sum x_{p} a_{n p}$ converges. Hence $A x$ is a complex sequence.

We next show that $A x \in S_{C}$. By Remark 1 we note that $\sum x_{p} a_{p}$ converges. Let $L$ be such that $\left|y_{p}\right|<L, p \geqq 1$. Suppose $\epsilon>0$. Let $Q$ be a positive integer such that $1 / 2^{Q}<\epsilon$ and if $p>Q$, then $\left|x_{p}\right|<\epsilon$ and $\left|\sum_{j=p}^{\infty} x_{j} a_{j}\right|<\epsilon$. Let $R$ be a positive integer such that if $t_{p}>R$, then $p>Q$. Let $N=Q+R$ and suppose $n>N$. If $t_{q} \leqq n<t_{q+1}$, then by use of (3) and (4), we have

$$
\begin{aligned}
\left|\sum_{p=1}^{\infty} x_{p} a_{n p}-\sum_{p=1}^{\infty} x_{p} a_{p}\right| \leqq & \left|\sum_{p=1}^{r_{q}} x_{p}\left(a_{n p}-a_{p}\right)\right|+\left|\sum_{p=r q+1}^{\infty} x_{p} a_{p}\right| \\
& +\left|\sum_{p=r q+1}^{r_{q+1}} x_{p} a_{n p}\right|+\left|\sum_{p=r q+1}^{r_{q+1}} x_{p} a_{n p}\right| \\
& +\left|\sum_{p=r q+2}^{\infty} x_{p} a_{n p}\right| \\
& <2 L / 2^{q+1}+\epsilon+2 k \epsilon+2 k \epsilon+2 L / 2^{q+1} \\
& <(4 L+1+4 k) \epsilon .
\end{aligned}
$$

Thus $A x \in S_{C}$, and we see that $A x$ converges to $\sum_{p=1}^{\infty} x_{p} a_{p}$. The theorem follows since we can take $y$ to be a null sequence not of bounded variation and then $x \in S_{C}-S_{B V}$.

REMARK 2. The proof of Theorem 1 can be modified slightly to prove the following statement:

If $A$ is a matrix which sums every sequence of bounded variation and $\alpha$ is an increasing sequence of positive integers, then there exists a subsequence $\beta$ of $\alpha$ such that if $\gamma$ is an increasing sequence of positive integers such that for some $m,\left\{\gamma_{p}\right\}_{p=m}^{\infty}$ is a subsequence of $\beta, y$ is a null sequence, and $F(y, \gamma)=x$, then $A x \in S_{C}$.

TheOREM 2. If $M$ is a countable set of matrices, each of which sums every sequence of bounded variation, then there exists a convergent sequence not of bounded variation which every matrix in $M$ sums.

Proof. The proof will be for the case that $M$ is infinite. Suppose $M=\left\{M_{1}, M_{2}, M_{3}, \cdots\right\}$. Let $\beta^{(1)}$ be an increasing sequence of positive integers which has the property with respect to $M_{1}$ which $\beta$ has with respect to $A$ in Remark 2 . Let $\beta^{(2)}$ be a subsequence of $\beta^{(1)}$ such that $\beta^{(2)}$ has the property with respect to $M_{2}$ which $\beta$ has with respect to $A$ in Remark 2. We continue the process to obtain $\beta^{(1)}, \beta^{(2)}$, $\beta^{(3)}, \ldots$ such that if $q$ is a positive integer, then $\beta^{(q+1)}$ is a subsequence of the increasing sequence $\beta^{(q)}$ of positive integers, and $\beta^{(q+1)}$ 
has the property with respect to $M_{q+1}$ which $\beta$ has with respect to $A$ in Remark 2. Let $\beta_{1}^{(0)}=\beta_{1}^{(1)}$ and let $\beta_{2}^{(0)}$ be the first integer greater than $\beta_{1}^{(0)}$ such that $\beta_{2}^{(0)}$ is a term of $\beta^{(2)}$. Let $\beta_{3}^{(0)}$ be the first integer greater than $\beta_{2}^{(0)}$ such that $\beta_{3}^{(0)}$ is a term of $\beta^{(3)}$. Continue the process. Suppose $y$ is a null sequence and $F\left(y, \beta^{(0)}\right)=x$. Let $n$ be a positive integer. Clearly there exists $m$ such that $\left\{\beta_{p}^{(0)}\right\}_{p=m}^{\infty}$ is a subsequence of $\beta^{(n)}$. Thus by Remark 2, $M_{n} x \in S_{C}$. In particular, if $y$ had been chosen as a null sequence not of bounded variation, then $x$ would have been a convergent sequence not of bounded variation. Thus the theorem follows.

We now show that in Theorem 2 we cannot replace the set $M$ with the set of all matrices which sum every sequence of bounded variation. To this end, suppose $\left\{b_{p}\right\} \in S_{C}-S_{B V}$. By a theorem of Hadamard [2], there exists $\left\{a_{p}\right\} \in S$ such that $\sum a_{p}$ converges and $\sum b_{p} a_{p}$ diverges. Let $A=\left(a_{p q}\right)$ be defined as follows: $a_{p q}=a_{q}$ if $p \geqq q, a_{p q}=0$ if $p<q$. Clearly $A$ satisfies (i), (ii), and (iii) of (1) and therefore sums every sequence of bounded variation, but $A$ does not sum $\left\{b_{p}\right\}$.

\section{REFERENCES}

1. J. P. Brannen, Concerning Hausdorff matrices and absolutely convergent sequences, Proc. Amer. Math. Soc. 15 (1964), 114-123.

2. J. Hadamard, Deux théorèmes d'Abel sur la convergence des séries, Acta Math. 27 (1903), 177-183.

3. B. Kuttner, On discontinuous Riesz means of type n, J. London Math. Soc. 37 (1962), 354-364.

4. K. Zeller, Über den perfekten Teil von Wirkfeldern, Math. Z. 64 (1956), 123-130.

5. — Vergleich des Abelverfahrens mit gewöhnlichen Matrixverfahren, Math. Ann. 131 (1956), 253-257.

North Texas State University 